\title{
Teoría del valor desde una perspectiva contable: una revisión bibliográfica
}

\author{
Theory of Value from an Accounting Perspective: \\ A Bibliographic Review
}

Lucas Ardila Jiménez, Karen Fonseca Niño, Anggy López León, Carolina Sánchez Ortiz, Johan Sánchez Lerma*

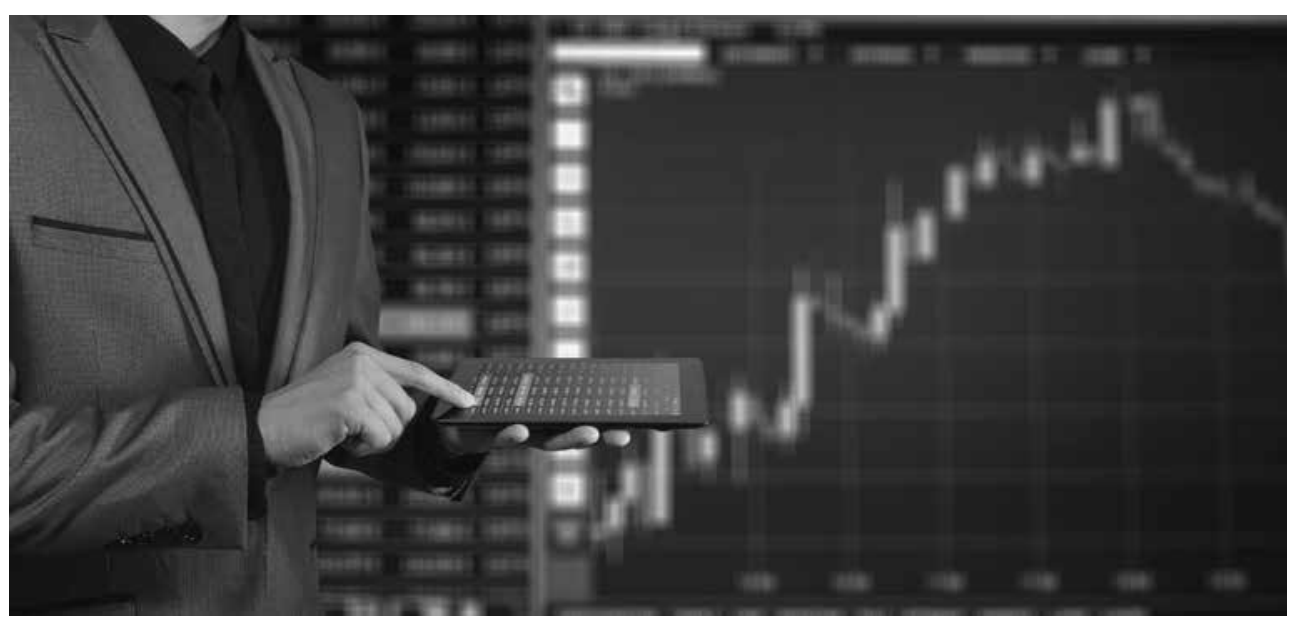

\section{Resumen}

En el siguiente documento se expone una breve búsqueda sistemática de literatura en teoría contable, la cual esté sujeta o aborde el debate de la teoría del valor. Se utilizan diversas bases de datos y la colaboración de profesores en teoría contable de la Universidad Nacional de Colombia para obtener los textos a analizar tanto en español como en inglés. En cada texto se analiza y se sintetiza el desarrollo del debate sobre la teoría del valor, buscando evidenciar si se ha logrado una estandarización de la valuación en la teoría contable por medio del análisis de las diferentes posturas de los autores trabajados en esta investigación.

Palabras clave: teoría, contabilidad, valor, valuación.

Citar este artículo como: Ardila, L., Fonseca, K., López, A. Sánchez, C. y Sánchez, J. (2020). Teoría del valor desde una perspectiva contable: una revisión bibliográfica. Revista Papeles, 11(22), 59-71.

Fecha de recibido: septiembre 15 de 2019 - Fecha de aceptación: noviembre 10 de 2019

* Estudiantes del curso de Metodología de la Investigación I de la Universidad Nacional de Colombia, sede Bogotá, durante el periodo 2019-II, dirigidos por el profesor Enrique Ferrer-Corredor. Correos electrónicos: Lucas Ardila Jiménez (lfardilaj@unal.edu.co), Karen Fonseca Niño (klfonsecan@unal. edu.co), Anggy López León (allopezl@unal.edu.co), Carolina Sánchez Ortiz (csanchezo@unal.edu. co), Johan Sánchez Lerma (jodsanchezle@unal.edu.co). 


\begin{abstract}
In the following document, we present a brief systematic search of literature on accounting theory, which is addressed or linked to the debate on theory of value. Several databases and the collaboration of professors in accounting theory from the Universidad Nacional de Colombia are used to obtain the texts to be analyzed in both Spanish and English. In each text, the development of the theory of value debate is analyzed and synthesized, seeking to evidence whether a standardization of valuation in accounting theory has been achieved through the analysis of the different positions of the authors working on this research.
\end{abstract}

Keywords: theory, accounting, value, valuation.

\title{
Problema investigativo
}

Desde los inicios de la economía como disciplina se ha discutido acerca de la teoría del valor, llevando a las primeras reflexiones sobre la comercialización 'justa'. Las perspectivas de la teoría del valor se han dividido en objetivas y subjetivas. "Las teorías objetivas sostienen que el valor está en las cosas, mientras que las subjetivas sostienen que el valor lo da el individuo" (Cachanosky, 1994); por lo tanto, los conceptos cambian de acuerdo con la posición o criterio del autor.

La teoría del valor ha sido un referente importante para las ciencias económicas, en especial para la contabilidad; no obstante, para esta disciplina ha sido necesario profundizar sobre la aplicación y utilidad de la teoría, haciendo posible, a través del tiempo, generar su propia discusión y perspectiva sobre la misma.

A partir de allí se hace preciso indagar en el siguiente debate o reflexión académica: ¿se ha logrado una uniformidad o estandarización de lo que se entiende por teoría del valor en la disciplina contable?

El objetivo de esta investigación es recopilar y realizar una clasificación de las diferentes perspectivas acerca de la teoría de valor en la disciplina contable. Se pretende buscar la relación existente entre las diferentes perspectivas y determinar la preponderancia de una línea de pensamiento sobre la teoría del valor en la disciplina contable.

\section{Ruta metodológica}

Esta investigación utilizó el enfoque cualitativo, ya que se realizó una revisión sistemática de literatura, utilizando portales y bases de datos tales como SCOPUS y Web of Science, por medio de relaciones entre los conceptos principales tanto en inglés como en español. Los textos encontrados se discriminaron por medio de la lectura de abstracts, identificando si verdaderamente se trataba el tema de teoría de valor y si pertenecían a la teoría contable.
Por otro lado, se consultó con profesores de la Facultad de Ciencias Económicas de la Universidad Nacional de Colombia, quienes tienen o han tenido relación con la teoría contable, buscando obtener diversos autores y bases teóricas para el desarrollo del proyecto. Cada texto fue analizado en lo relacionado con la teoría del valor, con el objetivo de la construcción de un estado del arte y la obtención de diversas conclusiones. 


\section{Teoría de valor y contabilidad}

La teoría del valor es un debate de cuestión ideológica en la teoría económica, el cual se extiende a otras disciplinas y teorías sociales, y que no tiene una única ni precisa definición. Esta tiene una relación directa con la relatividad histórica de las ideas y el pensamiento humano, siendo un debate que en su complejidad no ha tenido una conclusión. En esencia, esta teoría busca explicar el valor de cambio o los precios de los bienes y servicios.

Las tesis más conocidas de la teoría del valor en la ciencia económica son la teoría del valor-trabajo, la teoría del valor-intercambio, la teoría del valor de uso, la teoría monetaria del valor, la teoría del valor-poder y la teoría subjetiva del valor. Todas abordadas por diversos teóricos en la evolución de la discusión y la creación de nuevas corrientes económicas, lo cual convierte a la teoría del valor en un debate útil que se extiende más allá de la economía y que se puede encontrar en otras disciplinas.

Durante las últimas décadas distintos "matemáticos, filósofos y científicos sociales han dedicado muchas energías al análisis de conceptos vinculados con la medición y han extraído muchas sutilezas al respecto" (Mattessich, 2002, p. 147). Debido a estos análisis, se fueron incorporando paulatinamente las nociones de medición a los distintos saberes de acuerdo con sus necesidades, dando como resultado unas nuevas nociones agrupadas en algo conocido como la "Moderna Teoría de la Medición".

Para Stevens (1958, p. 383), “Ahora parece claro para nosotros que el proceso de medición es el proceso de organizar hechos y relaciones empíricas dentro de un modelo formal, un modelo tomado prestado por la matemática". Pero es bien sabido que la contabilidad ha descuidado este nuevo cuerpo de saber y que solo recientemente, por el esfuerzo de grandes autores, como Stevens, de implementar este nuevo conocimiento, se ha empezado a relacionarlo con su disciplina.
Es bien sabido que la contabilidad es un modelo determinístico dimensionado en dos escalas. Primero, un plan de cuentas que permite la clasificación bidimensional de hechos económicos y, segundo, unas ratios expresables en moneda corriente legal. La valuación, como un tema central de estudio en la contabilidad se define como el "procedimiento por el cual se asignan números a objetos o eventos de acuerdo con reglas con el objetivo de expresar preferencias con respecto a determinadas acciones" (Mattessich, 2002, p. 145). No es posible imaginar la existencia de una teoría de evaluación sin una teoría del valor; del mismo modo no es posible imaginar la existencia de una teoría del valor que no tenga consigo implícitamente nociones de valuación. Por lo tanto, es correcto afirmar que cada procedimiento de evaluación coherente nace de una teoría del valor.

En contabilidad, los procedimientos de valuación que más se utilizan son las escalas numéricas y brindan un rango de cada elemento. Así, el valor expresado por un número se supone que indica una preferencia por aquel objeto por parte de un individuo o un colectivo social.

La práctica contable trae consigo muchas imperfecciones en la valuación y, así mismo, en el desarrollo de su teoría. Mattessich (2002) plantea un ejemplo que demuestra esta situación:

Supongamos que nos ofrecen elegir entre un durazno maduro y una barra de chocolate blanco y elegimos el primero siempre que el propósito sea nuestro consumo individual del bien en cuestión. Podríamos, sin embargo, elegir la barra de chocolate si nuestra intención fuera dárselo al chico que está jugando a la vuelta de la esquina al que sabemos que le gusta dicha golosina (p. 148).

Este ejemplo muestra cómo el propósito influye en nuestra preferencia ante la presencia de muchos propósitos y circunstancias para 
el mismo objeto; así que, tanto la teoría como la práctica deben reconocer que tenemos que atribuir diferentes valores bajo condiciones distintas al mismo objeto.

La historia de las ciencias económicas, al igual que muchas otras ciencias, ilustra de una forma $u$ otra la diversidad de tratamientos que pueden darse al fenómeno del valor y su respectiva medición.

Desde los economistas preclásicos, pasando por la presentación de Adam Smith y la parcial exposición de David Ricardo, hasta la temprana y profunda visión de Hermann Heinrich Gossen, el problema del valor ha cambiado constantemente su aspecto en cada uno de ellos (Mattessich, 2002, p. 149).

Por poner un ejemplo de cambio en la concepción de valor, se tiene que hablar expresamente del "paradigma utilitarista de la contabilidad" (Dueñas, 2007, p. 145). Este paradigma está principalmente relacionado con el actuar de los agentes en un entorno complejo, llamado mercado, y que por su misma naturaleza exige que cada agente en el mercado tome la adecuada decisión en hechos particulares del momento. Aunque, como sabemos:

El simple hecho de tomar una decisión implica elegir entre múltiples opciones, lo que a fin de cuentas desemboca en que la decisión dependerá principalmente de una evaluación de que sobre determinado hecho o cosa realice el agente en el mercado (Dueñas, 2007, p. 146-148).

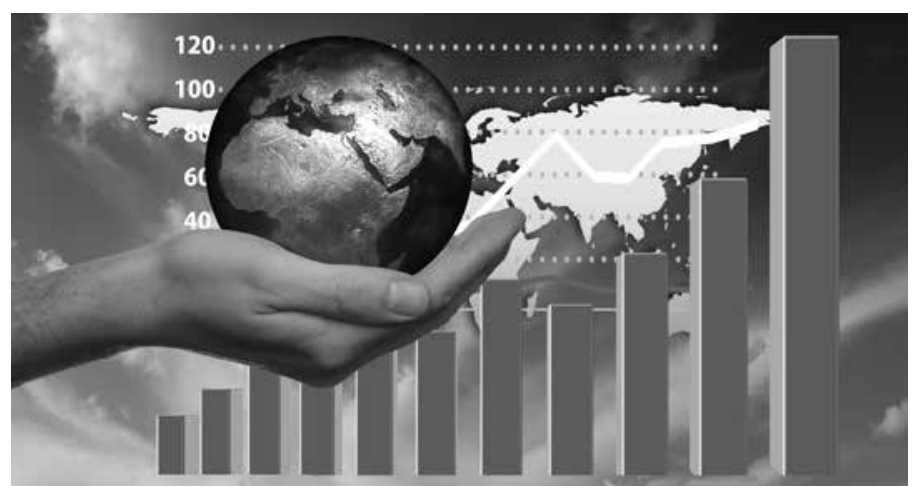

Los economistas clásicos desde un principio concebían el valor como una cualidad intrínseca de un bien. También se iniciaron estudios sobre la preocupación por el comportamiento del consumidor que llevó a los marginalistas a la concepción del concepto del valor como una relación entre un bien y un agente económico. Es aquí donde estos economistas determinan el valor a través de la satisfacción que el último incremento de este bien genera para el agente económico. Por su parte, los contables llevaron este debate a su campo de estudio, poniendo énfasis en el contexto en el que se expresa el valor; así se puede ver el valor como parte de un complejo sistema de conceptos entrelazados de distintas áreas y ciencias. Sin embargo, para llegar a este punto, la teoría contable ha debatido, adaptado y estandarizado la tarea de la "valuación de activos", dejando de un lado el debate de carácter filosófico de la teoría económica al utilizar métodos o modelos estandarizados para determinar el respectivo valor, métodos que abandonan la controversia de explicar el valor de cambio o precio de un bien y se adhieren a la normatividad presente en el contexto.

Igualmente, la globalización, el cambio en los mercados, el comercio internacional y la dependencia de economías abiertas de sus balanzas comerciales han generado una movilidad de capitales tanto reales como financieros que demandan información contable entendible sin importar el contexto, facilitando la toma de decisiones y disminuyendo la incertidumbre. Por ende, el valor y su medición ha quedado fuera de los temas de discusión entre contadores, gerentes, financieros y otros agentes del mercado, ya que la volatilidad y grandilocuencia del comercio internacional lo ha dejado supeditado a los estándares internacionales de información financiera, dando garantías informativas a los diferentes agentes del mercado.

No es una hipérbole afirmar que las condiciones de los mercados actuales son extremadamente diferentes a las de los 
mercados cuando la escuela clásica de economía, la escuela austriaca y los marginalistas, entre muchos otros, crearon y expusieron propuestas respecto a la teoría de valor. La apertura económica modificó estrictamente la manera en que se estudian las ciencias económicas, así como los modelos macroeconómicos predominantes, que tenían como supuestos que la economía era cerrada, los cuales quedaron obsoletos y pasaron a segundo plano; asimismo sucedió con lo referente a la teoría del valor.
No obstante, para llegar a esta estandarización en la disciplina contable, necesariamente se ha tenido que abordar directa o indirectamente la teoría del valor. ¿Por qué se valora de una manera $u$ otra los activos en contabilidad? ¿Qué línea de pensamiento predomina? Con una evolución de más de 500 años, la teoría contable ha abordado la teoría del valor desde su disciplina, aunque nunca ha profundizado en el debate filosófico, que, por el contrario, sí aborda la teoría económica dada su naturaleza disciplinar.

\section{Teoría del valor en la literatura contable}

\section{Richard Mattessich}

Richard Mattessich ${ }^{1}$ (2002) en su libro Contabilidad y métodos analíticos: medición y proyección del ingreso y la riqueza en la microeconomía y la macroeconomía, hace una recopilación de definiciones o aproximaciones de cuál es el verdadero significado de la contabilidad. A partir de análisis y ciertas reflexiones lo lleva a la siguiente definición:

La contabilidad es una disciplina que se ocupa de la descripción cuantitativa y de la proyección de la circulación de ingresos y de los agregados de riqueza a través de un método basado en el siguiente conjunto de supuestos básicos: valores monetarios, intervalos de tiempo, estructura, dualidad, agregabilidad, objetos económicos, inequidad de las acreencias monetarias, agentes económicos, entes, transacciones económicas, valuación, realización, clasificación, ingreso de datos, duración, extensión, materialidad y asignación (Mattessich, 2002, p. 18).

Los dieciocho supuestos básicos anteriormente planteados deberían hacer que esta

1 Economista de negocios austro-canadiense y profesor emérito de contabilidad de la Universidad de Columbia Británica. definición fuera lo suficientemente poderosa como para considerarla como fundamento de una teoría general y sistemática de la contabilidad.

Uno de estos supuestos básicos es el de la valuación, donde Mattessich hace una crítica de que muchos contadores y expertos en administración "rechazan la teoría subjetiva del valor de la economía tradicional como una base general de valuación para propósitos prácticos en todas las áreas de las ciencias económicas" (Mattessich, 2002, p. 158), y entonces muestra un interés particular por desarrollar métodos unificados de medición, que permitan a la disciplina contable estudiarlos y ponerlos en práctica a través de las diferentes normativas y lineamientos contables.

\section{Steven Toms}

Steven Toms (2010) en su artículo "Value, profit and risk: accounting and the resource based view of the firm" genera una reflexión frente a la aplicación de la teoría del valor trabajo de Karl Marx en las firmas que siguen el modelo RBV (visión basada en recursos). "The strategy literature, and especially the RBV, places emphasis on the role of human capital

2 Profesor de contabilidad y finanzas de la Universidad de York. 
in the creation of competitive advantage which at the same time poses problems for accountants in terms of total business and intangible asset valuation" (Toms, 2010). ${ }^{3}$ Con esto, Toms hace referencia a que una aplicación directa de la teoría del valor trabajo en la organización $y$, especialmente en su recurso humano, crea una ventaja competitiva sostenible (SCA), dándole mayor relevancia a los trabajadores y convirtiendo el recurso humano en un activo intangible de alto valor, pero, a la vez, generando dificultades para el contador en cuanto a la manera en que se registra este activo intangible, y la manera que se controla y monitorea por medio de las finanzas dicha creación de valor. Así, vemos cómo Toms hace referencia a un escenario específico en un modelo organizacional aplicando la teoría del valor-trabajo y los retos que este trae para los contadores.

\section{Steven Toms y Cliff Bowman}

Steven Toms y Cliff Bowman ${ }^{4}$ (2008) elaboraron un artículo denominado "Accounting For Competitive Advantage: The ResourceBased View Of The Firm And The Labour Theory of Value"; allí se mencionan los retos de clasificación, sistematización y medición del valor-trabajo en los trabajadores de una organización. Con la misma línea argumentativa de la visión basada en recursos y su aplicación de teoría del valor.

Capital is not in itself sufficient to explain the distribution of surplus between workers, managers and shareholders. The outcome depends also on the processes of accountability, which begin

3 "La literatura sobre estrategia, y especialmente el RBV, pone énfasis en el papel del capital humano en la creación de ventajas competitivas, lo que al mismo tiempo plantea problemas a los contadores en términos de valoración total del negocio y de los activos intangibles" (traducción propia).

4 Profesor de gestión estratégica de la Universidad de Cranfield. with valorisation in the labour process. Here the management accounting system ascribes homogenous monetary values to heterogeneous outputs from the service delivery or process. Because the process is less easily observable in the case of embedded capital, the probability of asymmetry between the social value of the labour expended and the realised monetary amount increases (Toms y Bowman, $2008)^{5}$.

Con la anterior referencia, los autores mencionan el conflicto contable frente a la valorización del proceso productivo de los diferentes trabajadores en las diferentes jerarquías dentro de la firma. Afirman que los contables adscriben valores monetarios homogéneos a los resultados o las salidas del proceso productivo de los trabajadores, las cuales tienen intrínsecos valores monetarios heterogéneos. Esto tiene similitud con el problema marxista de la transformación, en el cual existe un amplio debate que ha sido abordado por economistas como Engels, Veblen, Loria, Sombart, Pareto y el austriaco BöhmBawerk (Boundi, 2018). El tema de cómo los precios directos se derivan de los precios de producción ha creado controversia entre la literatura marxista, pero los planteamientos no suelen salir del análisis filosófico y económico, desconociendo que un análisis contable

5 "El capital no es en sí mismo suficiente para explicar la distribución del superávit entre los trabajadores, los directivos y los accionistas. El resultado depende también de los procesos de rendición de cuentas, que comienzan con la valorización en el proceso laboral. En este caso, el sistema de contabilidad de gestión atribuye valores monetarios homogéneos a los resultados heterogéneos de la prestación de servicios o del proceso. Dado que el proceso es menos fácil de observar en el caso del capital incorporado, aumenta la probabilidad de que haya asimetría entre el valor social del trabajo gastado y el importe monetario realizado" (traducción propia). 
es de grandilocuente utilidad. Más adelante, en este documento se menciona al autor Rob Bryer, quien aborda y resuelve el problema de la transformación desde el análisis contable.

\section{Beata Sadowska y Adam Lulek}

Beata Sadowska ${ }^{6}$ y Adam Lulek ${ }^{7}$ (2016) utilizan la teoría del valor-trabajo como fundamento para crear un esquema que explica la ruta principal y tarea principal de la teoría contable. Establecen que esta ruta es la medición de la emisión de datos característicos, lo cual permite prever la evolución de fenómenos y tener una herramienta de transformación de la realidad. "It reflects the reality, transposing processes to the financial dimension" (Sadowska y Lulek, 2016) ${ }^{8}$. Así, la teoría contable posee la tarea de reflejar la realidad por medio de los registros financieros, lo cual genera un reto mucho mayor en lo relacionado con la valuación, pasando de la teoría del valor a la teoría de la medición en la disciplina contable. Los autores exponen cómo el dilema de la teoría del valor ha estado presente como objeto de estudio desde las raíces de la filosofía y la historia del pensamiento económico.

Value has become a concept of philosophy in the second half of the nineteenth century. Earlier, the word "good" was used instead of the term "value". The first mention of the value can be found in the writings of Heraclitus of Ephesus from the sixth and fifth centuries before Christ, who distinguished the value of relating it to people, goods and services. The value

6 Doctora de la Facultad de Administración y Economía de Servicios de la Universidad de Szczecin.

7 Doctor de la Facultad de Administración y Economía de Servicios de la Universidad de Szczecin.

8 "Refleja la realidad, transponiendo los procesos a la dimensión financiera" (traducción propia). was measured using their morals, and the value of goods and services expressed their utility. The nature of value and its measurement have been a source of interest of Plato and Aristotle. Plato was a supporter of the objective and spontaneous value, which stemmed from the quality and characteristics of the evaluated units. Aristotle believed that the value is a result of human needs, which justifies the statement that changed things should be comparable with the standard reckoning. A contribution to science concerning the value, measurement and valuation also brought Protagoras, who lived in the fifth century BC. He made a statement: "man is the measure of all things" that led to the assertion that the values are the result of subjective experience and are dependent on the relationship to someone or something. Aristotle also dealt with the theory of measurement, starting from the premise that the best results are achieved when the measurement on impairment is made or the growth of a phenomenon is examined (Sadowska y Lulek, 2016) ${ }^{9}$.

9 "El valor se ha convertido en un concepto de filosofía en la segunda mitad del siglo XIX. Anteriormente, se utilizaba la palabra "bien" en lugar del término "valor". La primera mención del valor se encuentra en los escritos de Heráclito de Éfeso de los siglos VI y V a.C., quien distinguió el valor de relacionarlo con personas, bienes y servicios. El valor se medía por su moral, y el valor de los bienes y servicios expresaba su utilidad. La naturaleza del valor y su medición han sido una fuente de interés de Platón y Aristóteles. Platón era partidario del valor objetivo y espontáneo, que se derivaba de la calidad y las características de las unidades evaluadas. Aristóteles creía que el valor es el resultado de las necesidades humanas, lo que justifica la afirmación de que las cosas cambiadas deben ser comparables con el cálculo estándar. Una contribución a la ciencia en relación con el valor, la medición y la valoración también trajo a Protágoras, que 
Tabla 1. Escalas de medición con ejemplos de aplicación en contabilidad

\begin{tabular}{|l|l|}
\hline Escala nominal & $\begin{array}{l}\text { Eso del número de cuento (dígito) como el nombre de la clasificación de la cuenta. } \\
\text { Se utiliza el grupo que representa al activo dentro del estado financiero para medir } \\
\text { su valor. }\end{array}$ \\
\hline Escala ordinal & $\begin{array}{l}\text { Patrón de comparaciones. Por ejemplo: "falta de liquidez", "baja rotación de } \\
\text { inventarios". } \\
\text { Se crean patrones de comparación, tales como la velocidad con la cual el activo se } \\
\text { puede convertir en efectivo o la velocidad en la cual se mueven los inventarios. }\end{array}$ \\
\hline Escala de intervalos & $\begin{array}{l}\text { Costo estándar de contabilidad. La determinación del costo estándar es arbitraria y } \\
\text { sus derivados se puede medir. } \\
\text { Determina el valor por medio del costo estándar contable, el cual suele ser arbitra- } \\
\text { rio y se puede relacionar más directamente con la teoría subjetiva del valor de la } \\
\text { escuela austriaca de economía. }\end{array}$ \\
\hline Escala proporcional & $\begin{array}{l}\text { Estudio de los cambios en el valor en diferentes momentos, lo quelleva a afirmar que } \\
\text { el nivel del componente de los activos fijos es dos veces mayor que el año pasado. } \\
\text { Determina el valor, por medio de estudios, del precio del activo en los diferentes } \\
\text { tiempos; así, por medio de promedios ponderados genera la valuación. }\end{array}$ \\
\hline
\end{tabular}

Fuente: Sadowska y Lulek (2016 [traducción propia]).

Por ende, su evolución teórica llega a un campo multidimensional en lo que compete a la contabilidad, la cual no desconoce las teorías y tesis anteriores, sino que, en función de estas, crea herramientas para que la discusión llegue a su disciplina. Se crean escalas de medición dividas en dimensiones dependiendo del tipo de activo que se deba medir. Una simplificación de la teoría de la medición se puede resumir con la tabla 1.

Aquí se evidencia que la aplicación de la teoría del valor no está determinada en ninguna corriente específica, sino, por el contrario, varía dependiendo de la situación y las características del activo. Por ende, con

vivió en el siglo V a.C. Él hizo una declaración: "el hombre es la medida de todas las cosas" que llevó a la afirmación de que los valores son el resultado de la experiencia subjetiva y dependen de la relación con alguien o algo. Aristóteles también se ocupó de la teoría de la medición, partiendo de la premisa de que los mejores resultados se obtienen cuando se realiza la medición de la deficiencia o se examina el crecimiento de un fenómeno" (traducción propia). el trabajo de Beata Sadowska y Adam Lulek identificamos la evolución y adaptación de la teoría del valor en la disciplina contable, llegando a una propia teoría de la medición.

\section{Rob Bryer}

Rob Bryer ${ }^{10}$ (2014) contraargumenta el planteamiento de la aplicación de la teoría del valor en la teoría contable creando un documento de la aplicación de la teoría contable en la teoría del valor-trabajo de Karl Marx, haciendo referencia al "problema de la transformación de valores en precios de producción" que aborda en el tercer tomo de $E l$ Capital. Bryer expone cómo la aplicación contable es una herramienta eficaz para resolver el problema mencionado anteriormente.

Marx's accounting solution to the 'transformation problem' is that because the market price of all identical commodities is equal, for the labour theory of value to work the same must be true

10 Profesor emérito de contabilidad de la Universidad de Warwick, Inglaterra. 
of its components, socially demanded profit and socially necessary cost (Bryer, 2014) $)^{11}$.

Así, aplicando la teoría del valor y sus postulados, por medio de un razonamiento contable, se argumenta la igualdad entre el trabajo socialmente necesario monetizado en commodities y se supera el problema de la transformación.

Bryer establece que la contabilidad debería ser aplicada con mayor frecuencia por los teóricos marxistas, ya que puede ser muy útil para resolver controversias sobre la aplicación de Marx en el capitalismo moderno. Con el trabajo de Bryer tenemos un ejemplo de cómo en la literatura de teoría contable se expone una relación de complementariedad entre el uso de la contabilidad y la elaboración de los postulados marxistas en su teoría del valor.

\section{Pieter Willem Buys}

Pieter Willem Buys ${ }^{12}$ (2009) ha utilizado la teoría del valor desde su aspecto científico, apoyándose en las primeras bases del pensamiento económico desde los filósofos morales griegos hasta pensadores más contemporáneos para explicar la manera en que se aborda la valuación de activos en la contabilidad, hasta llegar a una contabilidad basada en el valor. En su artículo "Reflections on the value concept in accounting", publicado para la escuela de ciencias contables de la Universidad

11 "La solución contable de Marx al 'problema de la transformación' es que, como el precio de mercado de todas las mercancías idénticas es igual, para que la teoría del valor del trabajo funcione, lo mismo debe ocurrir con sus componentes, la ganancia socialmente exigida y el costo socialmente necesario" (traducción propia).

12 Filósofo contable sudafricano con doctorados en ciencias contables y en contabilidad de gestión, y con maestrías en ciencias económicas y en contabilidad de gestión. de Sudáfrica, Willem llega a sus conclusiones basándose en tres premisas: las diferencias en el valor de uso o cambio de los bienes, el deseo particular sobre un bien no únicamente basado en razones económicas sino en deseos sociales y morales, y el valor percibido en un objeto es directamente proporcional a la urgencia en el deseo de este.

Value theory essentially relates to the exchange ratio between two commodities or services. Seen in the light of accounting's objective of translating operational performances into financial terms, the absence of clear guidelines on the contextual concept of value can lead to the phenomena of different values for different purposes. Accounting debates on value interpretations, as well as on the methods and policies around value measurement, have considerable room for subjective value judgements (Willem, $2009)^{13}$.

En la anterior cita Willem afirma que la necesidad contable de una traducción de los resultados operaciones a términos financieros y la ausencia de lineamientos claros hace que la medición del valor esté sujeta al propósito del contador. "When we depart from the assumption that there is not a single correct application of the value concept, we see that value is governed by varying situations and

13 "La teoría del valor se relaciona esencialmente con la relación de intercambio entre dos productos o servicios. Visto a la luz del objetivo de la contabilidad de traducir los resultados operativos en términos financieros, la ausencia de directrices claras sobre el concepto contextual de valor puede conducir a fenómenos de valores diferentes para fines diferentes. Los debates contables sobre las interpretaciones de los valores, así como sobre los métodos y políticas en torno a la medición de los mismos, dejan un margen considerable para los juicios de valor subjetivos" (traducción propia). 
scenarios"14 (Willem, 2009). De esta manera, Willem nos da a entender que los objetivos de la contabilidad como la toma de decisiones, la comparación de hechos históricos y el reflejo de la realidad de la organización quedan sometidos por el oportunismo de los contadores y gestores. Teniendo en cuenta este argumento, desde el planteamiento de Willem, la contabilidad basada en el valor, así como el valor histórico, pierden veracidad y pueden ser tergiversados con sencillez.

\section{Aracely del Socorro y Martha Arias}

Aracely del Socorro ${ }^{15}$ y Martha Liliana $\operatorname{Arias}^{16}$ (2012) realizan una concepción acerca de lo que es el valor desde Aristóteles hasta la percepción del mismo en nuestros días, finalizando con la estandarización a través de una normatividad como lo son las NIIF. En primera medida, Aristóteles se convierte en pionero en hacer una diferenciación entre el valor de uso y el valor del cambio, "su énfasis está puesto en las distintas maneras de adquirir bienes que tienen las personas, una de las cuales es el intercambio" (Sánchez Serna y Arias Bello, 2012), primando una justicia entre el intercambio de bienes. San Agustín, por su parte, es mencionado en el texto que relaciona el valor con la percepción y sensibilidad del ser, es decir, se da una reflexión subjetiva de

14 "Cuando nos apartamos del supuesto de que no hay una sola aplicación correcta del concepto de valor, vemos que el valor se rige por diversas situaciones y escenarios" (traducción propia).

15 Contadora pública y licenciada en matemáticas de la Pontificia Universidad Javeriana. Máster en finanzas corporativas y negocios internacionales de la Universidad Católica de Ávila.

16 Contadora pública de la Pontificia Universidad Javeriana, MBA de la Universidad de los Andes y consultora de Contaduría General de la Nación. lo que el valor o el intercambio significa para cada individuo.

A partir de este punto en la historia, se presentan pensamientos que, si bien, son de distintas escuelas y con lapsos de años considerables, se puede denotar que los pensamientos convergen en el factor de producción y el valor determinado al objeto con base en ello. Se sigue resaltando el valor de la justicia, en el cobro y pago por dicho bien, pero se busca llevar de la mano el tiempo y trabajo invertido en la elaboración, producción o realización del producto o de la actividad. Es en este momento en que el precio, que no es igual a la valoración para estos pensadores, hace parte de la balanza, para equilibrar y compensar el trabajo, convirtiéndose de esta forma "como unidad de medida para el intercambio" (Sánchez Serna y Arias Bello, 2012).

Algo semejante a lo anteriormente mencionado ocurre con los economistas clásicos; durante el documento se menciona la percepción acerca del valor. En este caso, Adam Smith empieza hablar por primera vez del término "utilidad" como una expresión del valor de uso, para, de este modo, introducir el comportamiento del mercado, de acuerdo con la demanda y oferta de dicho bien. Sin embargo, se mantiene la distinción entre el valor de cambio y el valor de uso. En ese mismo orden de ideas, distintos economistas clásicos brindan su aporte a la teoría del valor, teniendo una concordancia con escuelas anteriores, pero especificando más en aspectos clave como los factores de producción y los distintos términos que hacen posible el concepto de salario y la valoración de este.

Ahora bien, en la actualidad, con la regulación de las NIIF, "según los Estándares Internacionales de Información Financiera, uno de los criterios básicos para el reconocimiento de una partida en los estados financieros es que tenga un costo o valor que se pueda medir con fiabilidad" (Sánchez Serna y Arias Bello, 2012). Este valor debe ser estimado, pero esta estimación debe llevar consigo una valoración de su uso y con base 
en ello dar una valoración en el mercado. La NIIF 13 evidencia dos premisas según el análisis de las autoras: una premisa de uso y una premisa de intercambio; no obstante, ambas están relacionadas con el precio del mercado.

Las autoras, a través de este artículo, realizan un recorrido sobre la percepción o concepción del valor desde épocas remotas, en donde inicialmente intentaban responder a preguntas de justicia y ética, como es mencionado en el texto. La evolución de estos conceptos de valor permitió llegar a un acuerdo normativo para la medida del valor de cada bien, tanto tangible como intangible.

\section{Nelson Javier Dueñas}

En la literatura contable también se ha relacionado la teoría subjetiva del valor con la disciplina. Es el caso de Dueñas ${ }^{17}$ (2007), quien realiza una revisión de la evolución del concepto de valor en economía para abordar desde la teoría subjetiva del valor la forma en que los individuos eligen y valoran objetos y sucesos de acuerdo con sus emociones, para finalmente exponer las razones sobre por qué la teoría subjetiva del valor no es aplicada en la contabilidad.

Sus argumentos inician con una revisión de las teorías evaluativas y emocionales, y el papel que estas desempeñan en las acciones humanas; de aquí se concluye que una persona, al valorar una cosa, incluye en dicho proceso sus emociones.
Posteriormente, Dueñas (2007) expone los diferentes conceptos de valor en la economía, el valor de uso y el valor de cambio. En el primero, la cantidad de trabajo que hay en la producción de una mercancía es lo que le da un valor, y en el valor de cambio, una mercancía dada recurre a otra para poder expresar su valor, siempre que se considere un cambio equivalente.

El valor de uso es lo que sustenta la teoría subjetiva del valor $y$, a su vez, esta es la base de la ley de utilidad marginal. Los individuos buscan satisfacer sus necesidades mediante la maximización de los beneficios; la palabra que debe llamar la atención es satisfacción, ya que es la manifestación de las emociones del individuo. En realidad, no hay una asignación de valor a los objetos sino a la satisfacción y utilidad que estos le brindan al individuo.

Dueñas (2007) afirma que la disciplina contable no adoptó la teoría subjetiva del valor porque no le sirve, dado que las organizaciones nunca valoran a través del costo marginal, ya que hallar su propia función de producción es complejo. La contabilidad debe asignar un valor numérico a cada hecho o transacción económica, por lo cual una utilidad ordinal, la cual depende del grado de satisfacción, no es viable para la disciplina.

Finalmente, el autor señala que la disciplina contable no debe limitarse a la valoración a través del costo histórico, como se ha venido haciendo; en su lugar, debe empezar una búsqueda de nuevos enfoques para la valuación.

\section{Conclusiones}

En un ambiente económico, valorar los objetos y las situaciones es fundamental. Una de las principales tareas de la contabilidad es hacer una asignación de valores monetarios, ya

17 MBA en Universidad de Concordia, contador público de Universidad Nacional de Colombia y miembro del Observatorio Latinoamericano en Contabilidad. sea con base en una razón objetiva o subjetiva; por tal razón, la teoría del valor ha tenido que evolucionar a la par con la disciplina, llegando a lo que actualmente se conoce como la teoría de la medición.

La práctica contable se desarrolla de distintas formas dependiendo del lugar, la normatividad e, incluso, el tipo de 
organización; sin embargo, mantiene siempre unos supuestos y reglas generales. Por lo tanto, la teoría del valor no ha tenido solo que evolucionar a través de autores y teorías que intentan explicar su aplicación en la práctica, sino que por sí misma se ha ido adaptando a las necesidades de los usuarios de la información financiera.

En economía existen diversas líneas de pensamiento que a lo largo de los años han estudiado la teoría del valor. Esto ha servido de base para que diferentes autores de la disciplina contable desarrollen sus propias ideas y explicaciones sobre el uso de esta teoría en la contabilidad.

La disciplina contable ha intentado direccionar, unificar y formalizar los conceptos relacionados con la teoría del valor a través de diferentes exponentes y por medio de regulaciones internacionales, como lo son las Normas Internacionales de Información Financiera. Sin embargo, los avances en el estudio de la teoría del valor son pocos y la mayoría de ellos se remiten a hacer una recopilación de lo dicho por economistas y filósofos, buscando respaldar o justificar los métodos empleados en la práctica contable, para medir o hacer valoraciones.

El término de valor empleado en las distintas áreas de las ciencias económicas fue, es y seguirá siendo objeto de estudio por su gran complejidad al momento de ser analizado, indistintamente del punto de vista del que se aborde, bien sea objetivo o subjetivo. En contabilidad existe un compromiso social que debe propender por satisfacer el interés general de los usuarios; por lo tanto, debe generar mejores métodos de valuación, trascendiendo los enfoques actuales para poder reflejar de la forma más adecuada la realidad económica presente en la cotidianidad.

Los análisis sobre el valor en contabilidad se pueden ver desde dos perspectivas. Una que intenta ser objetiva, creando parámetros y escalas de medición para así facilitar el ejercicio contable y, por otro lado, se tiene un enfoque subjetivo, el cual considera que el valor de cambio y el valor de uso pueden estar en función de la magnitud de la necesidad inmediata de un bien y ciertos deseos sociales y morales. Se infiere que, de aplicarse esta última con rigurosidad en la práctica contable, puede ser tergiversada con facilidad la interpretación contable de una organización, mostrando en términos financieros una realidad muy diferente a la que puede reflejarse en términos operacionales; por tal razón, varios autores rechazan la teoría subjetiva del valor como una herramienta que ayude al contador en sus funciones y, en su lugar, se considera que dificulta su trabajo y que la contabilidad no alcanzaría su objetivo más esencial, el de brindar información veraz a los usuarios. Dado lo anterior, es comprensible que varios autores empleen otros enfoques sobre la teoría del valor que sí logran mantener a la contabilidad dentro de unos parámetros de confiabilidad y certeza.

El objetivo principal de los autores analizados no es precisamente la generación de una nueva teoría del valor desde la perspectiva contable; en su lugar, buscan explicar cómo esta, traída desde la economía, se adapta y se desarrolla en la práctica contable. Estos autores los podemos agrupar y organizar en diferentes líneas de pensamiento que reflejan la afinidad de determinada de la teoría del valor para sus planteamientos contables. Los autores Mattessich, Willem Buys, del Socorro y Arias pueden ser clasificados como partidarios de las teorías del valor de uso y valor de intercambio. Toms, Bowman, Sadowska, Lulek y Bryer se muestran más afines con la teoría del valor trabajo. Finalmente, el autor Dueñas, puede ser clasificado como crítico de la teoría subjetiva del valor y afirma que dicha teoría es bilateral a la práctica contable.

Teniendo en cuenta lo anterior, se generan las siguientes reflexiones. El 40\% de los autores concuerda con la teoría del valor de uso y valor de cambio como mecanismos para tratar de alcanzar la objetividad demandada de la presentación de estados contables. 
El $50 \%$ concuerda con los postulados y los principios de la teoría del valor-trabajo, principalmente tratando de extrapolarla y llevar sus principios a una teoría de la medición en sí, la cual genera diferentes escalas para la valoración contable. Por otro lado, Dueñas, representando el porcentaje restante, argumenta que la teoría del subjetiva del valor es lo que predomina en la medición de activos en la contabilidad, pero propone una evolución de esta teoría, para llegar a métodos de valuación de menor sesgo. Así, la mayoría de los autores señalan el riesgo de la implementación de la teoría subjetiva del valor y su fundamento en la utilidad marginal en la práctica contable por su posible tergiversación de la realidad organizacional desde lo contable.

Por último, teniendo en cuenta los postulados de los diferentes autores utilizados, se considera que se ha tratado de llegar a una estandarización frente a la teoría del valor en teoría contable, como producto de una pugna frente al sesgo que produce la subjetividad situacional aplicada a la valoración de activos en el reflejo financiero de la realidad organizacional. Así, aceptando principios de las diferentes teorías, se ha buscado una evolución tanto ideológica en el debate filosófico como frente a la normatividad que regula las prácticas de la profesión.

\section{Referencias}

Boundi, F. (2018). El problema de la transformación de valores en precios de producción. Una revisión de literatura en torno a las soluciones de Marx, Bortkiewicz-Winternitz y Morishima. Ensayos de Economía, 28(52), 101-126. doi: 10.15446/ede.v28n52.72275

Bowman, C., y Toms, S. (2008). Accounting for Competitive Advantage: The ResourceBased View of the Firm and The Labour Theory of Value. Critical Perspectives on Accounting, 21(3), 183-194. doi:10.1016/j. cpa.2008.09.010

Bryer, R. (2014). Marx's accounting solution to the 'transformation problem'. Warwick Business School Working Paper.

Buys, P. (2009). Reflections on the value concept in accounting. Koers, 74(3), 495-517. doi:10.4102/koers.v74i3.135

Cachanosky, J. C. (1994). Historia de las teorías del valor y del precio. Parte I. Revista Libertas, 20.

Dueñas Gil, N. J. (2007). La teoría subjetiva del valor en contabilidad: comentarios sobre la valoración con base en las emociones. Innovar, 17(30), 145-152.

Mattessich, R. (2002). Contabilidad y métodos analíticos; medición y proyección del ingreso y la riqueza en la microeconomía y macroeconomía. Buenos Aires: La ley.

Sánchez, A. y Arias, M. (2012). Concepción de valor y precio desde Aristóteles a los clásicos: una reflexión a la luz de las premisas de valoración de las Normas Internacionales de Información Financiera, NIIF. Cuadernos de Contabilidad, 13 (33), 433-462.

Sadowska, B. y Lulek, A. (2016). Measuring and valuation in accounting - theoretical basis and contemporary dilemmas. World Scientific News, 57, 247-256.

Stevens, S. (1958). Medida y Hombre. Science, 21, 383-409.

Toms, S. (2010). Value, profit and risk: accounting and the resource-based view of the firm. Accounting, Auditing \& Accountability Journal, 23(5), 647-670. doi:10. 1108/09513571011054927 